\title{
FTIR study of CPD photolyase with substrate in single strand DNA
}

\author{
I M. Mahaputra Wijaya ${ }^{1}$, Tatsuya Iwata ${ }^{1,2}$, Junpei Yamamoto ${ }^{3}$, Kenichi Hitomi ${ }^{4}$, Shigenori Iwai ${ }^{3}$, \\ Elizabeth D. Getzoff ${ }^{4}$ and Hideki Kandori ${ }^{1,2}$ \\ ${ }^{1}$ Department of Frontier Materials, Nagoya Institute of Technology, Showa-ku, Nagoya 466-8555, Japan \\ ${ }^{2}$ OptoBioTechnology Research Center, Nagoya Institute of Technology, Showa-ku, Nagoya 466-8555, Japan \\ ${ }^{3}$ Graduate School of Engineering Science, Osaka University, Toyonaka, Osaka 560-8531, Japan \\ ${ }^{4}$ Department of Integrative Structural and Computational Biology and The Skaggs Institute for Chemical Biology, The Scripps Research \\ Institute, La Jolla, CA 92037
}

Received November 17, 2014; accepted December 17, 2014

Photolyases (PHRs) utilize near UV/blue light to specifically repair the major photoproducts (PPs) of UV-induced damaged DNA. The cyclobutane pyrimidine dimer (CPD)PHR binds flavin adenine dinucleotide (FAD) as a cofactor and repairs CPD lesions in double-stranded DNA. To understand the activation and repair mechanism of CPDPHR, we applied light-induced difference Fourier transform infrared (FTIR) spectroscopy to CPD-PHR, whose signals were identified by use of isotope-labeling. To further investigate the enzymatic function, here we study the activation and repair mechanism of CPD-PHR with the substrate in single strand DNA, and the obtained FTIR spectra are compared with those in double-stranded DNA, the natural substrate. The difference spectra of photoactivation, the fully-reduced $\left(\mathrm{FADH}^{-}\right)$minus semiquinone (FADH') spectra, are almost identical in the presence of single strand and double-stranded DNA, except for slight spectral modification in the amide-I region. On the other hand, the difference spectra of photorepair were highly substrate dependent. Strong bands of the $C=O$ stretch $\left(1,720-1,690 \mathrm{~cm}^{-1}\right)$ and phosphate vibrations $\left(1,090-1,060 \mathrm{~cm}^{-1}\right)$ of double-stranded DNA may have disappeared in the case of single strand DNA. However, an isotope-labeled enzyme study revealed that spectral features upon DNA repair are similar between both substrates, and the main reason for the apparent spectral difference originates from structural flexibility of DNA after repair.

Correspondence author: Hideki Kandori, Department of Frontier Materials, Nagoya Institute of Technology, Showa-ku, Nagoya 466-8555, Japan.

e-mail: kandori@nitech.ac.jp
Key words: DNA repair, light-induced difference FTIR spectra, FAD, hydrogen bond

UV light emitted from the sun causes damage to DNA by initiating the photoisomerization of two adjacent pyrimidine bases. This reaction typically results in the formation of either a cyclobutane pyrimidine dimer (CPD) or a pyrimidine(6-4)-pyrimidone photoproduct ((6-4) PP). Many organisms, including all three kingdoms of life, have a rapid response DNA-repair enzyme that utilizes near-UV/blue light for the removal of lesions. This enzyme, named photolyase (PHR), can restore two native pyrimidines from a single UV-induced photoproduct by electron transfer from its light-activated cofactor. To date, two types of PHRs are known based on the specific lesions they repair: CPD- and (6-4) PHR [1,2].

PHRs bind flavin adenine dinucleotide (FAD) as a cofactor $[3,4]$. The fully reduced form of FAD $\left(\mathrm{FADH}^{-}\right)$is responsible for the DNA repair activity of PHR [5], where an electron is transferred from the excited $\mathrm{FADH}^{-}$to the DNA substrate in a light-dependent manner, leaving the cofactor in the neutral semiquinone radical state $\left(\mathrm{FADH}^{\circ}\right)$. Once the restoration of two pyrimidines is complete, an electron is transferred back from the repaired DNA to PHR, and the FAD cofactor is returned to its original fully reduced form [6]. When we prepared E. coli CPD-PHR in vitro, the enzyme contained $\mathrm{FADH}^{\cdot}$ [7], which can be converted to the active form $\left(\mathrm{FADH}^{-}\right)$by illumination with $>550 \mathrm{~nm}$ light [8]. Although the crystal structures of CPD-PHR have been obtained both for substrate-unbound and -bound forms, the 
enzyme dynamics following FAD activation ( $\mathrm{FADH}^{-}$formation) and conformational changes of DNA-enzyme complex upon photorepair remains poorly understood.

Our group thus initiated studies of CPD-PHR as well as (6-4) PHR by using light-induced difference FTIR spectroscopy, for which measuring conditions, including sample preparation method, were established [8-10]. The resulting difference spectra indicated structural alterations to FAD, side-chains and the peptide backbone of the enzyme, as well as the substrate [8-13]. In the case of CPD-PHR, we recently reported a comprehensive analysis of isotope labeled ${ }^{13} \mathrm{C}$ and ${ }^{15} \mathrm{~N}$ using a special E. coli strain, resulting in the successful separation of FAD, substrate and enzyme vibrational signals [13].

In this paper, we further extended the FTIR study of CPDPHR by measuring the activation and repair spectra for CPD in single strand DNA (ssDNA), but not in double-stranded DNA (dsDNA). In the case of the native substrate (CPD in dsDNA), the CPD photoproduct flips out upon binding to PHR, and then refolds into the DNA duplex after repair [14]. In contrast, ssDNA possesses a more flexible structure than dsDNA, although there is likely to be a common mechanism for binding, activation and repair. We thus compared lightinduced FTIR spectra of activation and repair between ssDNA and dsDNA.

\section{Materials and Methods}

E. coli CPD-PHR was prepared as described previously [8]. As the MTHF (5,10-methylenetetrahydrofolate) cofactor binds loosely to the wild-type enzyme of $E$. coli CPDPHR, we used the E109A mutant of CPD-PHR, which lacks MTHF binding, for our analysis [15]. This enzyme can still be photoreduced and has CPD repair activity. Uniform ${ }^{13} \mathrm{C}$ labeling was performed as described, where all vibrational signals of FAD and enzyme were ${ }^{13} \mathrm{C}$-labeled [13], while only substrate was unlabeled in the present study. The CPD module was synthesized as described previously [16], and was incorporated at the center (underlined) of the ssDNA oligonucleotide carrying the following sequence:

\section{5'-CGCGAATTGCGCCC-3'}

As the CPD-PHR sample complex was not amenable to the drying process required for FTIR measurements, unlike (6-4) PHR, a concentrated aqueous CPD-PHR sample was used for FTIR measurements as described previously [8]. To measure CPD-PHR with its substrate at a 1:1 stoichiometric composition by FTIR, $1 \mu \mathrm{L}$ of $2 \mathrm{mM} \mathrm{CPD}$ substrate in water was placed onto a $\mathrm{BaF}_{2}$ window $(18 \mathrm{~mm} \phi)$, and dried until the volume of CPD reached approximately less than $0.5 \mu \mathrm{L}$. Subsequently, $1 \mu \mathrm{L}$ of $2 \mathrm{mM} \mathrm{CPD-PHR}$ was added, and re-dried until the total volume became about $1 \mu \mathrm{L}$, so that the salt concentration in the buffer remained approximately unchanged during sample preparation. The samples were then sandwiched by another $\mathrm{BaF}_{2}$ window and sealed with Parafilm.
FTIR spectra were measured with a FTS-7000 (DIGILAB) equipped with an Optistat-DN cryostat and an ITC-4 temperature controller. Sample illumination was provided at $277 \mathrm{~K}$ by a $300 \mathrm{~W}$ xenon lamp (Max-302, ASAHI SPECTRA) with $>550 \mathrm{~nm}$ and $>390 \mathrm{~nm}$ filters for activation and repair, respectively [8]. For both ssDNA and dsDNA, all substrates are bound to CPD-PHR at a 1:1 stoichiometric composition in the dark. Under such conditions, photoactivation and phtorepair spectra are quantitatively comparable. The obtained difference FTIR spectra in the presence of CPD photoproduct in ssDNA were compared to those in the following dsDNA.

\section{5'-CGCGAATTGCGCCC-3' 3'-GCGCTTAACGCGGG-5'}

\section{Results and Discussion}

\section{Photoactivation and photorepair spectra of CPD-PHR} in the presence of CPD substrate in single strand DNA

Figure 1a compares light-induced difference FTIR spectra of CPD-PHR activation in the presence of ssDNA (red line) and dsDNA (black line) carrying a CPD lesion. The activation spectra correspond to the $\mathrm{FADH}^{-}$minus $\mathrm{FADH}^{*}$ spectra when illuminated at $>550 \mathrm{~nm}$. Both spectra are very similar to each other. Our previous study reported the difference spectra being similar, even in the absence of CPD substrate [8]. This indicates that spectral changes in Figure 1a originate from the chromophore (FAD) and the surrounding protein moiety, but not from the substrate (CPD) and the surrounding protein moiety. This is further supported by our recent isotope-labeling study [13]. For instance, large peaks at 1,532 (-) and 1,395 (+) $\mathrm{cm}^{-1}$ in Figure 1a are attributed to $\mathrm{FAD}$ vibrations, the ring I vibration and the $\mathrm{N} 1=\mathrm{C} 10 \mathrm{a}$ stretching or $\mathrm{N} 1-\mathrm{C} 10 \mathrm{a}$ and $\mathrm{N} 5-\mathrm{H}$ bending mode from $\mathrm{FADH}^{*}$ and the rocking mode of $\mathrm{H} 5$ from $\mathrm{FADH}^{-}$, respectively $[8,13,15]$.

In addition, the peaks at $1,719(-) / 1,710(+) \mathrm{cm}^{-1}$ are noteworthy. They only appeared in the presence of substrate in dsDNA [8]. The detailed isotope study revealed that the main contribution comes from the $\mathrm{C} 4=\mathrm{O}$ stretch of $\mathrm{CPD}$ substrate, whereas the $\mathrm{C}=\mathrm{O}$ stretch of a protonated carboxylic acid residue is also involved. E275 is the most likely candidate, and hydrogen-bonding alterations near the substrate and E275 are coupled to helical structural perturbation [13]. Figure 1a shows identical spectra for the 1,719 (-)/ $1,710(+) \mathrm{cm}^{-1}$ bands, suggesting that similar events take place with the CPD substrate in ssDNA. A small but reproducible difference was observed at 1,655-1,645 $\mathrm{cm}^{-1}$ between ssDNA (red curve) and dsDNA (black curve). As the frequency corresponds to the characteristic amide-I vibration of helices, the present observation suggests that hydrogen-bonding alterations near the substrate and E275 occur similarly between dsDNA and ssDNA, but helical structural perturbations somehow differ between them. 


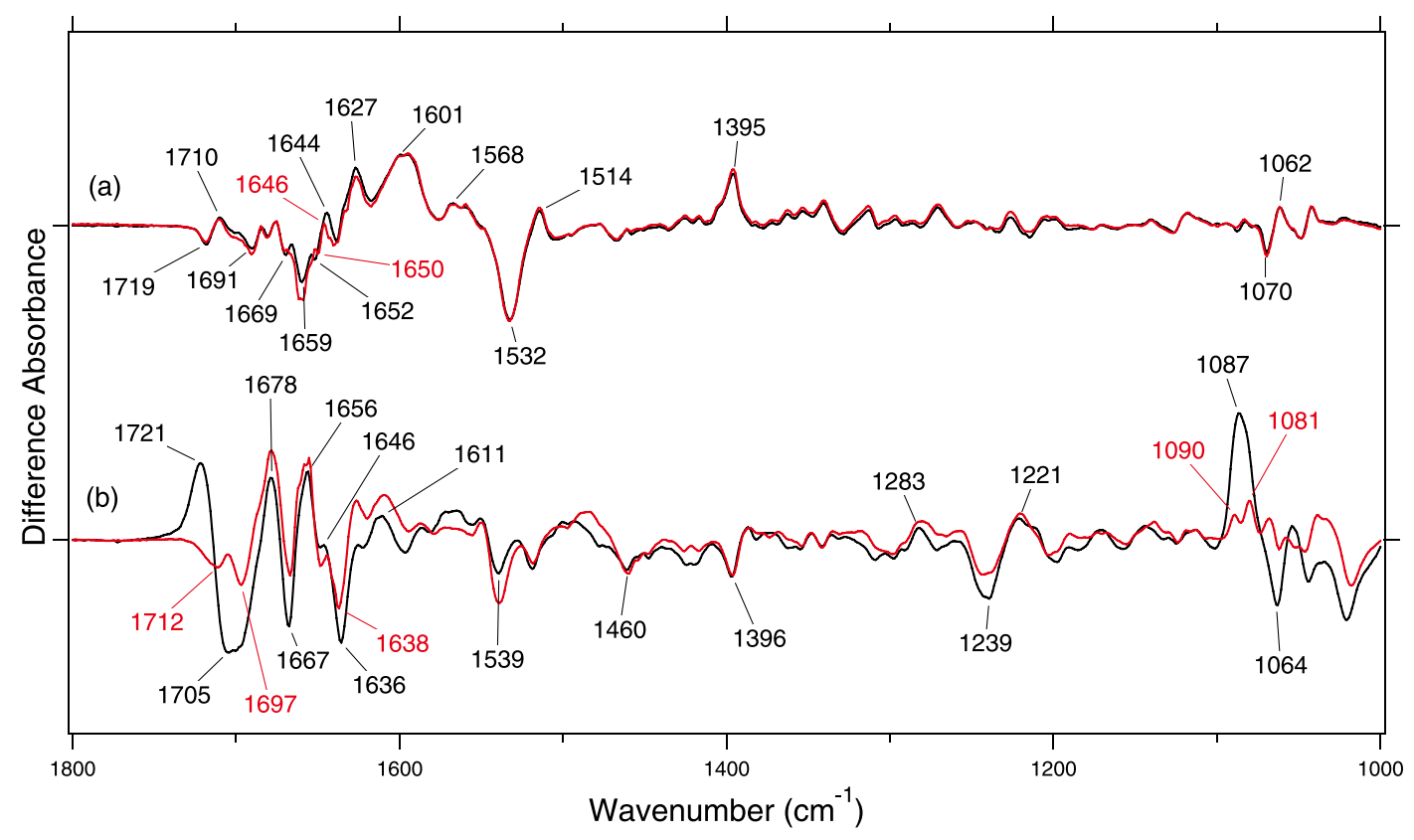

Figure 1 Light-induced difference FTIR spectra of CPD-PHR at $277 \mathrm{~K}$ showing photoactivation (a) and photorepair (b). (a) The semiquinone enzyme with the CPD substrate in single strand (red line) and double-stranded (black line) DNA was photoactivated by illumination with $>550 \mathrm{~nm}$ light for 2 min. (b) Photoactivated enzyme with the CPD substrate in single strand (red line) and double-stranded (black line) DNA was illuminated for photorepair with $>390 \mathrm{~nm}$ light for $2 \mathrm{~min}$. The molar ratio of enzyme to CPD was 1:1. Black spectra are reproduced from Wijaya et al. [8]. One division of the $y$-axis corresponds to 0.01 absorbance units.

Figure $1 \mathrm{~b}$ compares light-induced difference FTIR spectra of CPD-PHR repair between ssDNA (red line) and dsDNA (black line) carrying a CPD lesion. Our previous study showed that illumination of the fully reduced form $\left(\mathrm{FADH}^{-}\right)$ of PHR using $>390 \mathrm{~nm}$ excitation light resulted in no spectral changes without substrate, but rather in a clear spectral difference with substrate, which corresponds to the photorepair of CPD substrate into normal dsDNA [8]. This is also the case for CPD substrate in ssDNA (red line in Fig. 1b). Nevertheless, interestingly, the obtained spectra were largely different between ssDNA (red line) and dsDNA (black line). This is in clear contrast to the activation spectra, which are very similar between ssDNA and dsDNA (Fig. 1a). The reason for such a large difference could be explained as follows. For the activation spectra (Fig. 1a), CPD substrate is bound to the enzyme before and after illumination, and the structure of the active center is similar. On the other hand, the repaired substrate is released from the enzyme for the repair spectra (Fig. 1b), indicating that the repaired ssDNA and dsDNA are folded into the most stable states in aqueous solution. In the case of dsDNA, a normal DNA duplex is formed after repair, but the ssDNA structure is more flexible. Such structural differences probably influenced the repair spectra. Observation of enzymatic turnover under the present experimental conditions [8] strongly supports this idea. It should be however noted that a detailed discussion can only be possible after the assignment of vibrations. We recently assigned vibrational bands in the activation and repair spectra of CPD-PHR [13], which is the case for dsDNA. Therefore, we next measured difference FTIR spectra of ${ }^{13} \mathrm{C}$-labeled CPD-PHR, which carries unlabeled CPD substrate in ssDNA, and the obtained spectra were compared with those of dsDNA.

\section{Vibrational assignment of the photoactivation spectra of CPD-PHR by ${ }^{13} \mathrm{C}$-labeling}

Figure 2a compares the activation spectra of unlabeled (red curve) and ${ }^{13} \mathrm{C}$-labeled (blue curve) enzyme in the presence of ssDNA carrying a CPD lesion. Vibrational modes of FAD and the protein moiety containing carbon exhibited an isotope shift, whereas no spectral shifts were expected for the vibrational modes of DNA. If vibrations of FAD and the protein moiety do not involve molecular motions of carbon, then they do not show an isotope shift. However, Figure 2a indicates that most vibrations exhibited an isotope shift. This is consistent with the fact that the activation spectra resemble those in the absence of CPD substrate [8]. Such an interpretation is also the case for CPD-substrate in dsDNA (Fig. $2 b)$ reported recently [13].

Figure $2 \mathrm{a}$ and $\mathrm{b}$ show peaks at $1,719(-) / 1,710(+) \mathrm{cm}^{-1}$, which do not appear without substrate [8]. As they are located in the characteristic frequency of protonated carboxylic acids, its structural change was suggested in a previous paper $[8,13]$. If this were the case, then the bands should show a spectral down-shift by $\sim 40 \mathrm{~cm}^{-1}$. However, peaks are clearly present at $1,717(-) / 1,706(+) \mathrm{cm}^{-1}$, and this indicates 


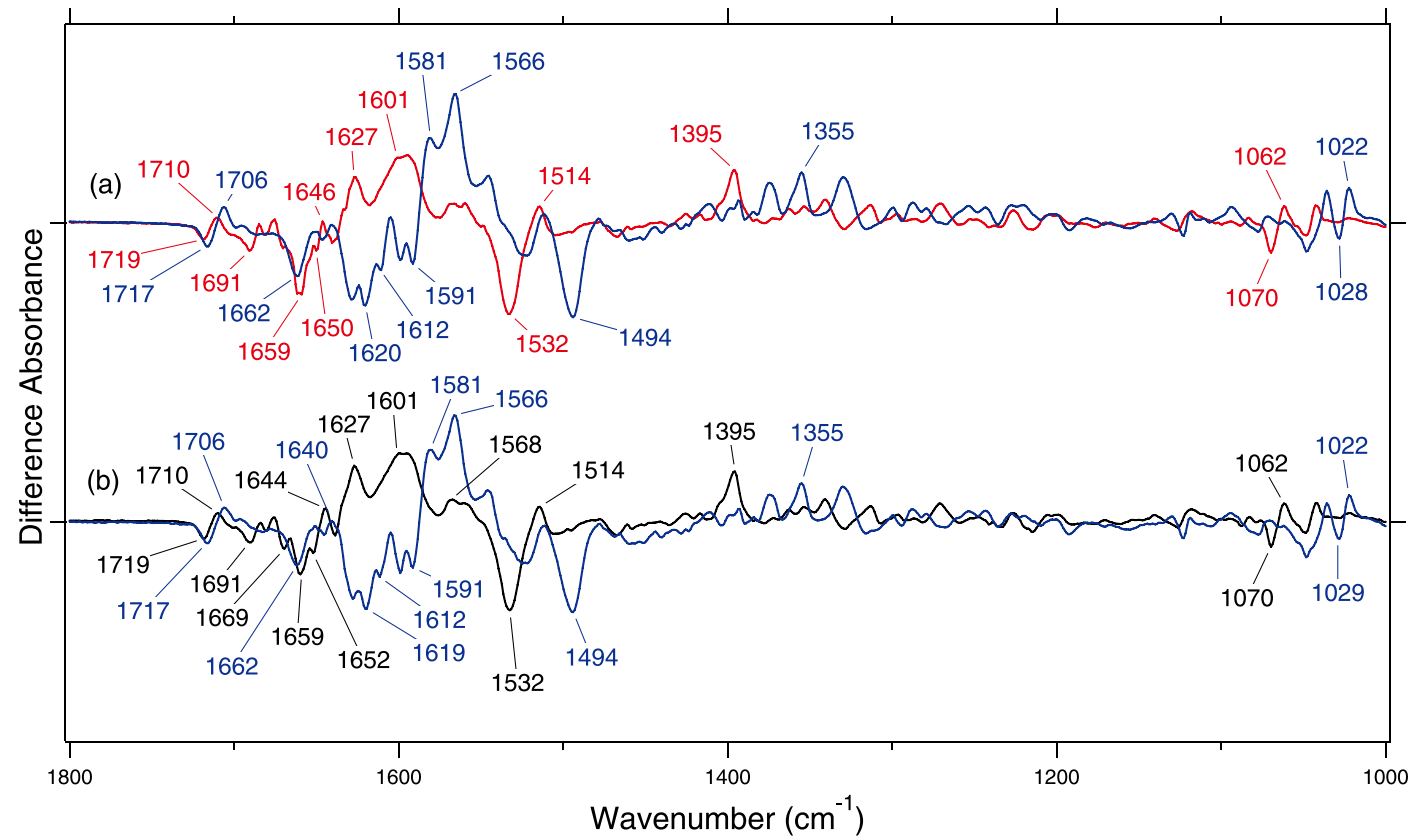

Figure $2{ }^{13} \mathrm{C}$ isotope effect on the light-induced difference FTIR spectra of CPD-PHR photoactivation. The semiquinone enzyme with the CPD substrate in single strand (a; red: unlabeled, blue: ${ }^{13} \mathrm{C}$ labeled) and double-stranded (b, black: unlabeled, blue: ${ }^{13} \mathrm{C}$ labeled) DNA was photoactivated by illumination with $>550 \mathrm{~nm}$ light for $2 \mathrm{~min}$ at $277 \mathrm{~K}$. In blue spectra, FAD and enzyme are ${ }^{13} \mathrm{C}$ labeled, while DNA substrate is not labeled. The spectra for double-stranded DNA are reproduced from Wijaya et al. [8,13]. One division of the y-axis corresponds to 0.01 absorbance units.

that the bands are attributable to CPD substrate (blue curves in Fig. $2 \mathrm{a}$ and $\mathrm{b}$ ). Then, the down-shift by $2-4 \mathrm{~cm}^{-1}$ suggests the involvement of protein vibrations in this frequency region. Our recent study, which used dsDNA, suggested that the $\mathrm{C}=\mathrm{O}$ stretch of a protonated carboxylic acid residue results in peaks at $1,714(+) / 1,705(-) \mathrm{cm}^{-1}$, where the hydrogen bond is strong for the neutral semiquinone radical $\left(\mathrm{FADH}^{\circ}\right)$, and moderate for the fully reduced form $\left(\mathrm{FADH}^{-}\right)$ [13]. E275 is the most likely candidate for the protonated carboxylic acid, and the molecular event is common between ssDNA and dsDNA.

\section{Vibrational assignment of the photorepair spectra of CPD-PHR by ${ }^{13} \mathrm{C}$-labeling}

In Figure $1 b$, the greatest difference between ssDNA (red curve) and dsDNA (black curve) was seen at 1,750 $1,690 \mathrm{~cm}^{-1}$ and at $1,090-1,060 \mathrm{~cm}^{-1}$. The former is the frequency region of $\mathrm{C}=\mathrm{O}$ stretches of $\mathrm{DNA}$ and protonated carboxylic acids of enzyme [13]. In the case of dsDNA, large bands at 1,721 (+)/1,705 (-) $\mathrm{cm}^{-1}$ (black curve in Fig. 1b) were assigned as the $\mathrm{C} 4=\mathrm{O}$ stretch of $\mathrm{CPD}$, while a small signal of protonated carboxylic acid was involved. In contrast, only negative peaks were observed at 1,712 and $1,697 \mathrm{~cm}^{-1}$ for ssDNA (red curve in Fig. 1b). Strong peaks at 1,087 (+)/1,064 (-) $\mathrm{cm}^{-1}$ for dsDNA (black curve in Fig. 1b) were assigned as the symmetric vibration of the DNA phosphate backbone [13]. Such strong peaks disappeared in ssDNA (red curve in Fig. 1b). Here, the isotope effect on ssDNA was studied, and the results were compared with those for dsDNA.

Figure 3a compares the repair spectra of unlabeled (red curve) and ${ }^{13} \mathrm{C}$-labeled (blue curve) enzyme in the presence of ssDNA carrying a CPD lesion. Interestingly, blue spectra showed a positive peak at $1,711 \mathrm{~cm}^{-1}$ and a negative peak at $1,694 \mathrm{~cm}^{-1}$ while red spectra only possessed negative signals at this frequency. This complicated spectral feature can be interpreted as follows. First, the bands at 1,711 (+)/1,694 (-) $\mathrm{cm}^{-1}$ for ${ }^{13} \mathrm{C}$-labeled enzyme (blue curve) originate from the $\mathrm{C}=\mathrm{O}$ stretch of ssDNA. Second, the negative peaks at 1,712 and $1,697 \mathrm{~cm}^{-1}$ for unlabeled enzyme (red curve) come from the enzyme.

The pure contribution of the protein moiety at $>1,690 \mathrm{~cm}^{-1}$ for FAD activation and DNA repair processes can be obtained by subtracting the blue curve from red curve in Figure 2 and 3. The corresponding double difference spectra for FAD activation and DNA repair at $>1,690 \mathrm{~cm}^{-1}$ are shown in Figure $4 \mathrm{a}$ and $4 \mathrm{~b}$, respectively (see Fig. S1 for the 1,800 $1,000 \mathrm{~cm}^{-1}$ region). The double difference spectra of the FAD activation in the presence of ssDNA (red curve in Fig. 4a) are similar to those with dsDNA (black curve in Fig. 4a). This suggests similar events happening between ssDNA and dsDNA, which we proposed for dsDNA previously [13]. Meanwhile the double difference spectra for DNA repair show negative peaks at 1,711 and $1,699 \mathrm{~cm}^{-1}$ (red curve in Fig. 4b). Such a spectral feature at this frequency region is common for dsDNA (black curve in Fig. 4b), suggesting that E275 deprotonation occurs despite difference between ssDNA and dsDNA substrate. 


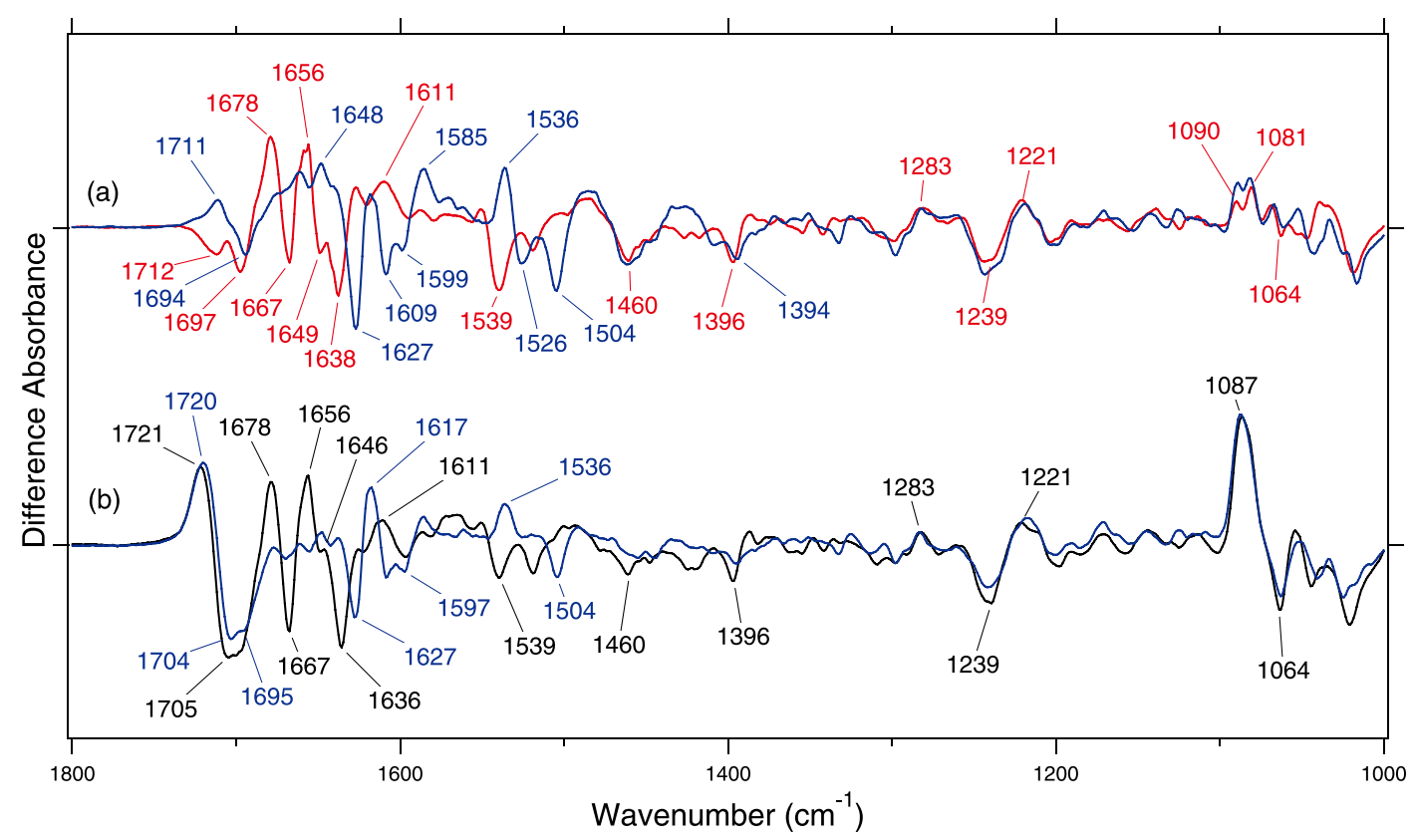

Figure $3{ }^{13} \mathrm{C}$ isotope effect on the light-induced difference FTIR spectra of CPD-PHR photorepair. Photoactivated enzyme with the CPD substrate in single strand (a; red: unlabeled, blue: ${ }^{13} \mathrm{C}$ labeled) and double-stranded (b, black: unlabeled, blue: ${ }^{13} \mathrm{C}$ labeled) DNA was illuminated for photorepair with $>390 \mathrm{~nm}$ light for 2 min at $277 \mathrm{~K}$. In blue spectra, FAD and enzyme are ${ }^{13} \mathrm{C}$ labeled, while DNA substrate is not labeled. The spectra for double-stranded DNA are reproduced from Wijaya et al. $[8,13]$. One division of the y-axis corresponds to 0.01 absorbance units.
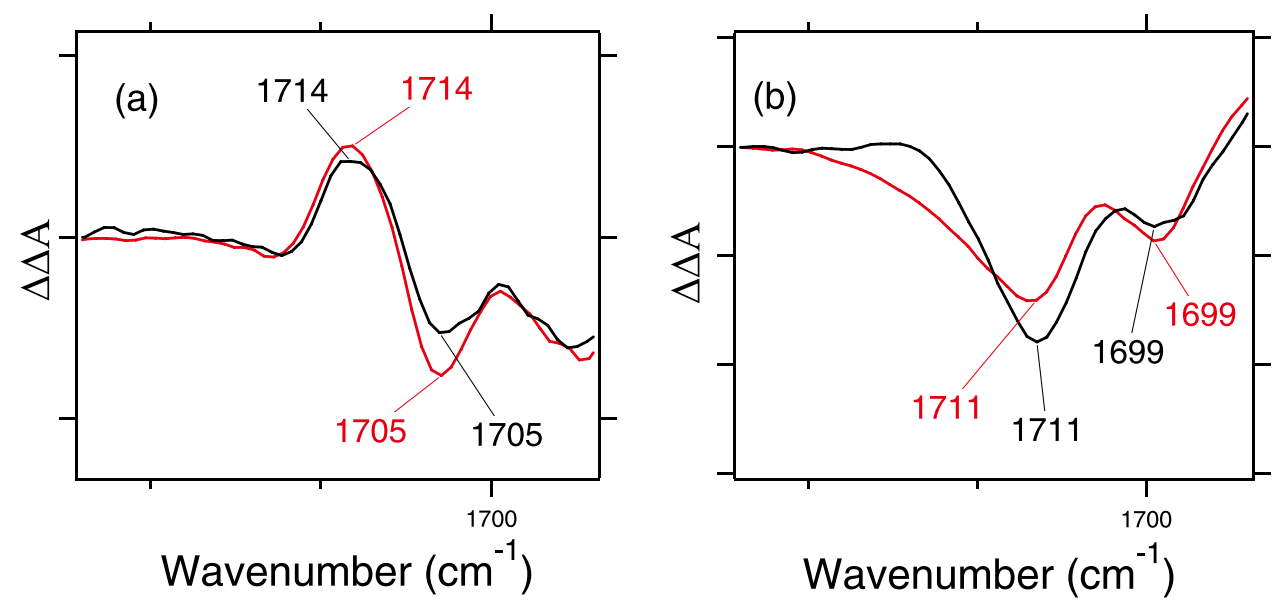

Figure 4 Double difference spectra showing contribution of enzyme in the 1,725-1,680 $\mathrm{cm}^{-1}$ region upon FAD activation (a) and DNA repair (b). Red and black curves represent the results for single-strand and double-stranded DNA, respectively. The double difference spectra for double-stranded DNA (black) are reproduced from Wijaya et al. [13].

It is thus possible to conclude that similar signals for repair between ssDNA and dsDNA exist in the 1,750$1,690 \mathrm{~cm}^{-1}$ region, although the repair spectra differ significantly from each other (Fig. 1b). The $\mathrm{C}=\mathrm{O}$ stretches of bound CPD substrate appear at 1,694 and $\sim 1,700 \mathrm{~cm}^{-1}$ for ssDNA and dsDNA, respectively, while the $\mathrm{C}=\mathrm{O}$ stretches of repaired thymine appear at 1,711 and $1,721 \mathrm{~cm}^{-1}$ for ssDNA and dsDNA, respectively. Protein vibrations appear at 1,711 and $1,694 \mathrm{~cm}^{-1}$ for both ssDNA and dsDNA, and in a previous paper, we suggested a possibility that the negative $1,711 \mathrm{~cm}^{-1}$ band is the deprotonation signal of E275 after the repair of CPD substrate in dsDNA [13]. A similar event occurs for the substrate in ssDNA. The apparent spectral difference between ssDNA and dsDNA originates from the amplitude of the $\mathrm{C}=\mathrm{O}$ stretch of DNA, where dsDNA (blue curve in Fig. 3b) shows a $>5$-times larger amplitude than ssDNA (blue curve in Fig. 3a). The reason for such a difference is unclear at present, although we infer a correlation 
with the structure of DNA after repair. In the case of dsDNA, the repaired thymine dimer is refolded into the DNA duplex, in which each thymine forms hydrogen bonds with adenine, leading to the unique frequency of the $\mathrm{C}=\mathrm{O}$ stretch $\left(1,721 \mathrm{~cm}^{-1}\right)$. In contrast, the structure of ssDNA is much more flexible after repair, in which each thymine has no clear counterpart, and possibly forms hydrogen bonds with water molecules. Consequently, the frequencies of the $\mathrm{C}=\mathrm{O}$ groups are distributed largely at $1,720-1,690 \mathrm{~cm}^{-1}$, leading to smaller difference peaks for ssDNA by cancelling due to $\mathrm{C}=4$ of ssDNA were bound with water.

Another interesting aspect of the blue curves in Figure 3 is the spectral feature. ssDNA shows single negative and positive peaks at 1,694 and $1,711 \mathrm{~cm}^{-1}$, respectively (blue curves in Fig. 3a). The positive band of dsDNA is also a single peak at $1,721 \mathrm{~cm}^{-1}$, whereas the negative band has two peaks at 1,704 and $1,695 \mathrm{~cm}^{-1}$ (blue curve in Fig. 3b). This suggests that the $\mathrm{C}=\mathrm{O}$ groups of DNA are under a heterogeneous environment only for the protein-bound CPD substrate in dsDNA. A single positive peak is probably reasonable, as the repaired thymines are released into aqueous solution, where $\mathrm{C}=\mathrm{O}$ groups must be under a similar environment. In contrast, the different feature in negative bands between ssDNA and dsDNA is intriguing. This observation suggests that dsDNA is more organized than ssDNA in the binding pocket, where the enzyme distinguishes two $\mathrm{C}=\mathrm{O}$ groups at $5^{\prime}$ and $3^{\prime}$ sides only in dsDNA.

The amide-I vibration (typically $1,700-1,600 \mathrm{~cm}^{-1}$ ) monitors secondary structural alterations in difference FTIR spectra, in which the $\alpha$-helix exhibits a characteristic frequency at $1,660-1,650 \mathrm{~cm}^{-1}$. The photorepair spectra indicate bond cleavage of the CPD substrate, and the repaired DNA is released into aqueous solution. We showed the involvement of structural alteration of the $\alpha$-helix upon repair of the CPD substrate in dsDNA $[8,13]$. Figure $1 b$ shows similar spectral features for the CPD substrate in ssDNA. In fact, peak frequencies are very similar at 1,679 (+), 1,667 (-), 1,656 (+) and $1,638(-) \mathrm{cm}^{-1}$, while amplitude is smaller in ssDNA than in dsDNA. It should be noted that the protein structure of the positive signal is identical between repair of ssDNA and dsDNA, indicating the same free enzyme state conformations after releasing the substrate. Therefore, the spectral difference in the amide-I region originates from the negative signal, which represents the substrate-enzyme complex state between ssDNA and dsDNA. In the case of dsDNA, the CPD substrate must be flipped out of the DNA duplex, which would be the additional motion to substrate binding. Therefore, the straightforward interpretation is that the amide-I band of ssDNA (red curve in Fig. 1b) monitors the substrate binding signal, while the additional spectral component of dsDNA (black curve in Fig. 1b) originates from the result of additional motion of dsDNA, such as a flipping out motion.

Figures $1 \mathrm{~b}$ and 3 show large differences in the symmetric vibrational region of the phosphate backbone of DNA at $1,090-1,060 \mathrm{~cm}^{-1}$. In the case of dsDNA, strong bands at
$1,087(+) / 1,064(-) \mathrm{cm}^{-1}$ originate exclusively from the vibration of DNA, because they are insensitive to the isotope effect. Negative and positive signals reflect from the phosphate group in the enzyme-bound form and in the DNA duplex, respectively. This is supported by the fact that the same positive band appeared for the repair spectra of the (6-4) substrate [9]. In the DNA duplex, symmetric vibration of the phosphate backbone appears at $1,087 \mathrm{~cm}^{-1}$, while it appears at $1,064 \mathrm{~cm}^{-1}$ for the enzyme-bound form. These spectral features, which disappear almost completely from ssDNA (Fig. 1b), are also likely to be correlated with structural flexibility in ssDNA. Unlike the DNA duplex, ssDNA has a flexible structure with widely distributed phosphate frequencies. Regarding the lack of negative peaks, we suggest the presence of a band at $1,064 \mathrm{~cm}^{-1}$ even for ssDNA (Fig. 3a), but repaired DNA in the single strand form also possesses a band at the same frequency. As a consequence, symmetric vibrations of the phosphate backbone are canceled for ssDNA. Positive peaks at 1,090 and $1,081 \mathrm{~cm}^{-1}$ for ssDNA, which exhibits no isotope shifts (Fig. 3a), are likely to originate from phosphate vibrations of ssDNA after repair [17]. In contrast to the symmetric phosphate vibrations, antisymmetric phosphate vibrations at 1,239 (-)/1,221 (+) $\mathrm{cm}^{-1}$ are not so different between ssDNA (Fig. 3a) and dsDNA (Fig. 3b). This suggests that anti-symmetric vibrations are less sensitive to the phosphate backbone structure than symmetric vibrations.

\section{Conclusion}

This paper reports the difference FTIR spectra of CPDPHR bound to CPD substrate in ssDNA upon photoactivation and photorepair. The obtained spectra were compared with those in dsDNA. The difference spectra of photoactivation were almost identical in the presence of ssDNS and dsDNA, and the unique spectral feature with the substrate indicates a similar structural environment of the CPD substrate between ssDNA and dsDNA. It would be reasonable to expect few differences between them because the photoactivation spectra mainly monitors the redox change of the FAD chromophore from the neutral semiquinone radical $\left(\mathrm{FADH}^{\circ}\right)$ to the fully-reduced $\left(\mathrm{FADH}^{-}\right)$form. In contrast, a prominent difference in the photorepair spectra was observed between ssDNA and dsDNA, especially for the region of the $\mathrm{C}=\mathrm{O}$ stretch $\left(1,720-1,690 \mathrm{~cm}^{-1}\right)$ and phosphate symmetric vibrations $\left(1,090-1,060 \mathrm{~cm}^{-1}\right)$ of DNA. Strong peaks in dsDNA disappeared in ssDNA, but the isotope-labeled study of enzyme revealed that spectral features were similar between both substrate. Apparent spectral differences between ssDNA and dsDNA arise mainly from the complex structure before repair and DNA substrate structures after repair. This view is summarized in Figure 5. Detailed spectral analysis of ssDNA and dsDNA by using mutants will lead to better understanding of the molecular mechanism of photorepair, which is our future focus. 
(a)

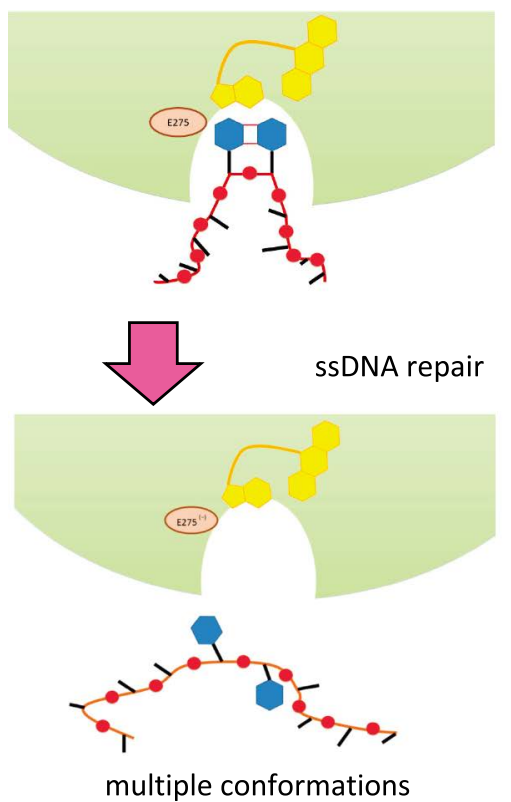

(b)

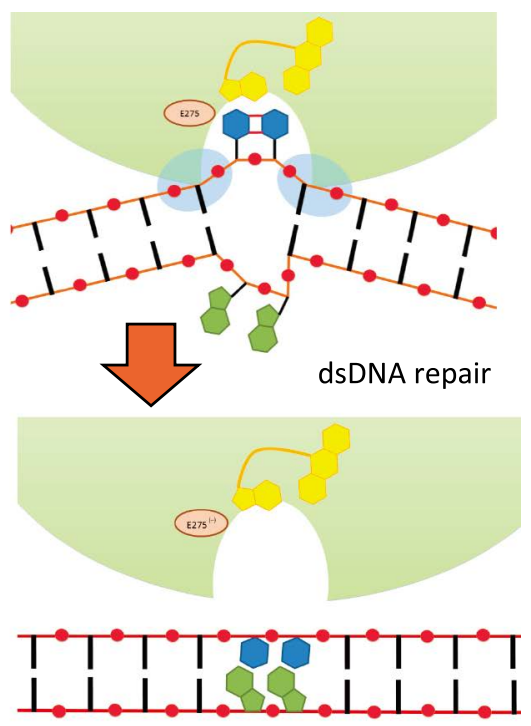

Figure 5 Schematic drawings of the repair processes of the CPD substrate in ssDNA (a) and dsDNA (b). The present study revealed similar binding and repair mechanism between ssDNA and dsDNA. On the other hand, structural flexibility for ssDNA after repair led to largely different spectra between ssDNA and dsDNA. In the figure, yellow, blue and green show FAD cofactor, thymine dimer (CPD) and paired adenines, respectively. Red circles represent phosphate group in DNA. After repair, ssDNA is refolded into multiple conformations, while dsDNA is refolded into a specific duplex conformation.

\section{Acknowledgment}

This work was supported by grants from the Japanese Ministry of Education, Culture, Sports, Science and Technology to H. K. (25104009).

\section{References}

[1] Sinha, R. P. \& Hader, D. P. UV-induced DNA damage and repair: a review. Photochem. Photobiol. Sci. 1, 225-236 (2002).

[2] Friedberg, E. C., Walker, G. C., Siede, W., Wood, R. D., Schultz, R. A. \& Ellenberger, T. DNA repair and mutagenesis, 2 nd edition, (ASM Press, ISBN: 978-55581-319-2.) (2006).

[3] Sancar, A. \& Sancar, G. B. Escherichia coli DNA photolyase is a flavoprotein. J. Mol. Biol. 172, 223-227 (1984).

[4] Todo, T., Kim, S. T., Hitomi, K., Otoshi, E., Inui, T., Morioka, H., Kobayashi, H., Ohtsuka, E., Toh, H. \& Ikenaga, M. Flavin adenine dinucleotide as a chromophore of the Xenopus (6-4) photolyase. Nucleic Acids Res. 25, 764-768 (1997).

[5] Payne, G., Heelis, P. F., Rohrs B. R. \& Sancar, A. The active form of Escherichia coli DNA photolyase contains a fully reduced flavin and not a flavin radical, both in vivo and in vitro. Biochemistry 26, 7121-7127 (1987).

[6] Sancar, A. Structure and function of DNA photolyase and cryptochrome blue-light photoreceptors. Chem. Rev. 103, 22032237 (2003).

[7] Jorns, M. S., Sancar, G. B. \& Sancar, A. Identification of a neutral flavin radical and characterization of a second chromophore in Escherichia coli DNA photolyase. Biochemistry 23, 2673-2679 (1984).

[8] Wijaya, I M., Zhang, Y., Iwata, T., Yamamoto, J., Hitomi, K.,
Iwai, S., Getzoff, E. D. \& Kandori, H. Detection of distinct $\alpha$-helical rearrangements of cyclobutane pyrimidine dimer photolyase upon substrate binding by Fourier transform infrared spectroscopy. Biochemistry 52, 1019-1027 (2013).

[9] Zhang, Y., Iwata, T., Yamamoto, J., Hitomi, K., Iwai, S., Todo, T., Getzoff, E. D. \& Kandori, H. FTIR study of light dependent activation and DNA repair processes of (6-4) photolyase. Biochemistry 50, 3591-3598 (2011).

[10] Yamada, D. \& Kandori, H. FTIR spectroscopy of flavin-binding photoreceptors. Methods Mol. Biol. 1146, 361-376 (2014).

[11] Zhang, Y., Yamamoto, J., Yamada, D., Iwata, T., Hitomi, K., Todo, T., Getzoff, E. D., Iwai, S. \& Kandori, H. Substrate assignment of the (6-4) photolyase reaction by FTIR spectroscopy. J. Phys. Chem. Lett. 2, 2774-2777 (2011).

[12] Yamada, D., Zhang, Y., Iwata, T., Hitomi, K., Getzoff, E. D. \& Kandori, H. Biochemistry 51, 5774-5783 (2012).

[13] Wijaya, I M., Iwata, T., Yamamoto, J., Hitomi, K., Iwai, S., Getzoff, E. D., Kennis, J. T., Mathes, T. \& Kandori, H. FAD chromophore charge controls the conformation of CPDphotolyase $\alpha$-helices. Biochemistry 53, 5864-5875 (2014).

[14] Berg, B. J. V. \& Sancar, G. B. Evidence for dinucleotide flipping by DNA Photolyase. J. Biol. Chem. 32, 20276-20284 (1998).

[15] Schleicher, E., Hessling, B., Illarionova, V., Bacher, A., Weber, S., Richter, G. \& Gerwert, K. Light-induced reactions of Escherichia coli DNA photolyase monitored by Fourier transform infrared spectroscopy. FEBS J. 272, 1855-1866 (2005).

[16] Murata, T., Iwai, S. \& Ohtsuka, E. Synthesis and characterization of a substrate for $\mathrm{T} 4$ endonuclease $\mathrm{V}$ containing a phosphorodithioate linkage at the thymine dimer site. Nucleic Acids Res. 18, 7279-7286 (1990).

[17] Taillandier, E. \& Liquier, J. Infrared spectroscopy of DNA. Methods Enzymol. 211, 307-335 (1992). 